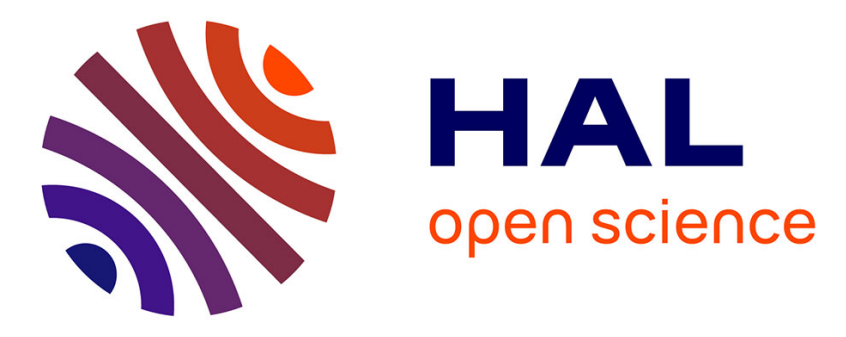

\title{
Saturation of multiplexed volume Bragg grating recording
}

S. Kaim, S. Mokhov, I. Divliansky, Vadim Smirnov, Julien Lumeau, B. Ya. Zel'Dovich, Leonid B. Glebov

\section{- To cite this version:}

S. Kaim, S. Mokhov, I. Divliansky, Vadim Smirnov, Julien Lumeau, et al.. Saturation of multiplexed volume Bragg grating recording. Journal of the Optical Society of America. A, Optics and image science, 2015, 32 (1), pp.22-27. hal-01274390

\section{HAL Id: hal-01274390 \\ https://hal.science/hal-01274390}

Submitted on 13 Mar 2019

HAL is a multi-disciplinary open access archive for the deposit and dissemination of scientific research documents, whether they are published or not. The documents may come from teaching and research institutions in France or abroad, or from public or private research centers.
L'archive ouverte pluridisciplinaire HAL, est destinée au dépôt et à la diffusion de documents scientifiques de niveau recherche, publiés ou non, émanant des établissements d'enseignement et de recherche français ou étrangers, des laboratoires publics ou privés. 


\title{
Saturation of Multiplexed Volume Bragg Grating Recording
}

\author{
Sergiy Kaim, ${ }^{1}$ Sergiy Mokhov, ${ }^{1}$ Ivan Divliansky, ${ }^{1}$ Vadim Smirnov, ${ }^{2}$ Julien Lumeau, ${ }^{3}$ \\ Boris Ya. Zeldovich, ${ }^{1, *}$ and Leonid B. Glebov ${ }^{1}$ \\ ${ }^{1}$ CREOL, The College of Optics and Photonics, University of Central Florida, Orlando, FL 32816 \\ 2 OptiGrate Corp, 562 South Econ Circle, Oviedo, Florida 32765-4311 \\ ${ }^{3}$ Aix-Marseille Université, CNRS, Centrale Marseille, Institut Fresnel, UMR 7249, 13013 Marseille, France \\ *Corresponding author: boris@creol.ucf.edu
}

Received Month X, XXXX; revised Month X, XXXX; accepted Month X, XXXX; posted Month X, XXXX (Doc. ID XXXXX); published Month X, XXXX

\begin{abstract}
Recording of Volume Bragg Gratings (VBGs) in Photo-Thermo-Refractive glass is limited to maximum refractive index change about 0.002. We discuss various saturation curves and their influence on amplitudes of recorded gratings. Special attention is given to multiplexed VBGs aimed at recording of several gratings in the same volume. The best shape of saturation curve for production of strongest gratings is the threshold-type curve. Two photon absorption as mechanism of recording also allows increasing the strength of multiplexed VBGs.

OCIS codes: (050.7330) Volume gratings; (090.4220) Multiplex holography.
\end{abstract}

\section{Introduction}

Volume Bragg Gratings (VBGs) [1, 2] recorded in Photo-ThermoRefractive (PTR) glass constitute new set of optical elements. They are used for spectral combining of high-power laser beams [3-5], for stretching and compression of ultra-short pulses [6, 7], for mode stabilization of diode lasers [8], for passive coherent beam combining [9], and for narrow band filtering in different fields of spectroscopy [10, 11]. Many of these elements are multiplexed VBGs, i.e. they contain several gratings with different spatial frequencies. Amplitudes of recorded gratings, both single and multiplexed ones, i.e. spatial Fourier-components of recorded $\delta n(\mathbf{r})$, are limited due to the fact of saturation of refractive index change by value $\Delta n_{\max }$. In the case of PTR glass the value of $\left|\Delta n_{\max }\right|$ in the best condition of recording and thermal development of VGB is about $\left|\Delta n_{\max }\right| \approx 0.002$.

Recording several volume holograms in the same volume has been studied theoretically and experimentally in [12-14] and numerous other works, with the purpose of data storage. Those studies were aimed to maximize the number of recorded holograms. The requirements to diffraction efficiency of each individual grating were not very stringent: diffracted wave had to be detectable at the level of rather weak noise. Most of those data-storage works were discussing and implementing multiplexed recording in electro-optic photorefractive crystals. Those crystals do not have strong response to the pedestal of recording beam intensity [15], therefore only small spatial period (spatial AC) components of illumination at recording were accounted for. As a result, the mean square average amplitude of spatial AC-modulation grows as $\sqrt{N}$, where $N$ is the number of gratings with independent periods and independent phases [13].

The main application of PTR glass-based VBG, both single and multiplexed ones, is handling high-power beams, be they inside the laser cavity or outside it. Therefore the achievement of large diffraction efficiency via generating strong spatial Fouriercomponent of refractive index modulation (RIM) is of essence. Exposure of the medium via single-photon absorption of interference patterns of pair of coherent waves with identical intensities and identical polarization is assumed to be:

$$
\begin{gathered}
U(\boldsymbol{r})=0.5 U_{1} \cdot\left|\exp (i \mathbf{k r})+\exp \left[i\left(\mathbf{k}+\mathbf{q}_{1}\right) \mathbf{r}+i \varphi_{1}\right]\right|^{2}= \\
=U_{1}\left[1+\cos \left(\mathbf{q}_{1} \mathbf{r}+\varphi_{1}\right)\right] .
\end{gathered}
$$

The main feature of PTR-VBG is that the pedestal part (spatially uniform), $U_{1}$, of the exposure, counts with the same coefficient as the grating part (spatially periodic), $U_{1} \cos \left(\mathbf{q}_{1} \mathbf{r}+\varphi_{1}\right)$. For large degree of multiplexing, $N \gtrsim 4$ it is the saturation of recording response by sum of pedestals $\sum_{j} U_{j} \sim U_{1} N$, that is the crucial factor. Meanwhile, individual gratings, $U_{j} \cos \left(\mathbf{q}_{j} \mathbf{r}+\varphi_{j}\right)$, have random phases, and their r.m.s. amplitude grows as $U_{1} \sqrt{N}$.

In this work we study theoretically the influence of saturation on recorded VBG with account of the following factors:

1) Normalized shape $\rho(U)$ of the saturation curve, i.e. dependence of refractive index change upon total exposure $U(\mathbf{r})$ : $\delta n(\mathbf{r})=\Delta n_{\max } \cdot \rho(U(\mathbf{r}))$.

2) Number $N$ of individual gratings aimed to be recorded.

3) Spatially-average exposure $\langle U\rangle \approx U_{1} N$ due to pedestal.

\section{Shapes of saturation curve $\rho(U(r))=\delta n(r) / \Delta n_{\max }$}

Presented below seven different shapes $\rho(U)$ chosen for our study are normalized in such a way that $\rho(U \rightarrow \infty)=1$. Besides that, first five curves under consideration have the property $\rho(U \rightarrow 0)=1 \cdot U$. The latter condition just means the choice of units of exposure. Here are seven different shapes that we have considered:

$$
\rho_{\tanh }(U)=\tanh (U)
$$




$$
\begin{gathered}
\rho_{\text {arctan }}(U)=(2 / \pi) \arctan (\pi U / 2), \\
\rho_{\text {power }}(U)=U /(1+U), \\
\rho_{\text {exp }}(U)=1-\exp (-U), \\
\rho_{\text {line } 45}(U)=\left\{\begin{array}{ll}
U & \text { at } 0<U<1 \\
1 & \text { at } U>1
\end{array},\right. \\
\rho_{\text {line } 60}(U)=\left\{\begin{array}{cl}
0 & \text { at } 0<U<0.5 \\
2(U-0.5) & \text { at } 0.5<U<1, \\
1 & \text { at } U>1
\end{array},\right. \\
\rho_{\text {heaviside }}(U)=\left\{\begin{array}{ll}
0 & \text { at } 0<U<0.5 \\
1 & \text { at } U>0.5
\end{array},\right.
\end{gathered}
$$

By saturation curve $\rho(U)$ we denote function $\rho(U)$ in the relationship, that we assume in our model:

$$
\delta n(\mathbf{r})=\Delta n_{\max } \cdot \rho(U(\mathbf{r}))
$$

Figure 1 shows the graphs of those seven functions; we put them into two different pictures to reduce the clutter.
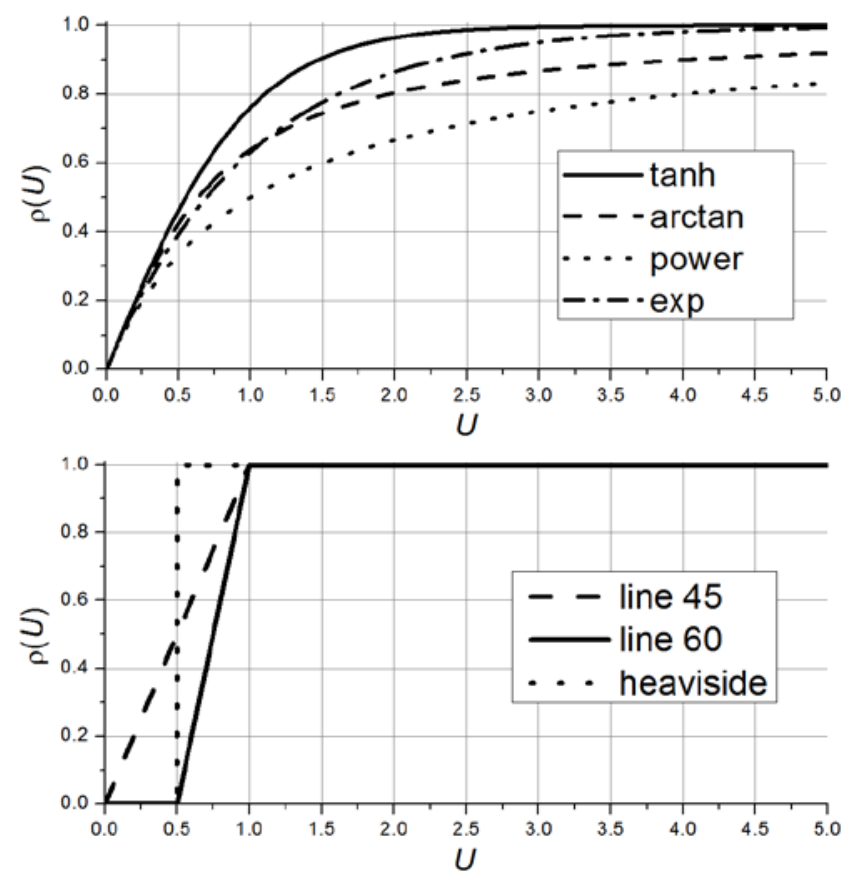

Fig. 1. Shapes of seven saturation curves under consideration.

It should be emphasized that there is no experimental evidence that the recording in Photo-Thermo-Refractive Glass (PTRG) satisfies any particular saturation law. Some theoretical models of PTRG recording and development point to power law, Eq. (3), as rather plausible variant [2]. However, the purpose of this modeling work is to make predictions following from all seven hypotheses, Eqs. (1-7) with the aim of subsequent comparison with future detailed experiments.

\section{Models of exposure}

We assume that an individual grating is recorded via interference pattern of two coherent waves. Intensity in such interference pattern is

$$
U_{j}\left(1+\cos \left(\mathbf{q}_{j} \mathbf{r}+\varphi_{j}\right)\right) .
$$

For single-photon absorption, the exposure by $N$ sequential interference patterns is

$$
U(\mathbf{r})=\sum_{j=1}^{N} U_{j}\left[1+\cos \left(\mathbf{q}_{j} \mathbf{r}+\varphi_{j}\right)\right]
$$

For definiteness we consider all $N$ intensities $U_{j}$ to be the same: $U_{j}=U_{1}$. Meanwhile the wave vectors $\mathbf{q}_{j}$ and phases $\varphi_{j}$ are considered statistically independent random quantities in our model.

For two-photon absorption of recording pattern of interference we take

$$
\begin{gathered}
U_{j}^{2}\left[1+\cos \left(\mathbf{q}_{j} \mathbf{r}+\varphi_{j}\right)\right]^{2} \equiv \\
\equiv U_{j}^{2}\left[1+2 \cos \left(\mathbf{q}_{j} \mathbf{r}+\varphi_{j}\right)+\frac{1}{2}+\frac{1}{2} \cos \left(2 \mathbf{q}_{j} \mathbf{r}+2 \varphi_{j}\right)\right] \equiv \\
\equiv \frac{3}{2} U_{j}{ }^{2}\left[1+\frac{4}{3} \cos \left(\mathbf{q}_{j} \mathbf{r}+\varphi_{j}\right)+\frac{1}{3} \cos \left(2 \mathbf{q}_{j} \mathbf{r}+2 \varphi_{j}\right)\right] .
\end{gathered}
$$

Note that relative modulation term at "right" spatial frequency has increased by factor 4/3. Besides that, second spatial harmonic appears in $U(\mathbf{r})$ for two-photon recording.

Figure 2 illustrates Eq. (1) and (3), and spatial profile of refractive index change for the case of $N=16$ independently recorded interference grating patterns with particular arbitrary values of $\mathbf{q}_{j}$ and $\varphi_{j}$. Spatially average exposure was chosen $U_{1} N=2.25$.
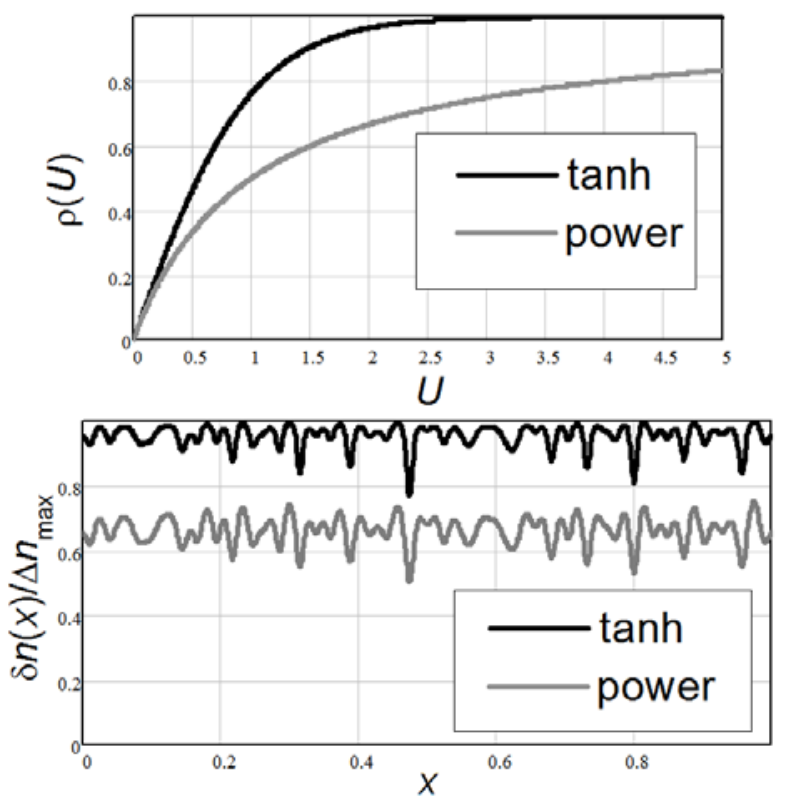

Fig. 2. a) Two saturation curves Eq. (1) and Eq. (3); b) Spatial profile of refractive index change of recording 16 multiplexed gratings.

\section{Fourier-component to be calculated}

By Fourier component of VBG we denote the quantity

$$
F_{j}=\frac{2}{V} \iiint \cos \left(\mathbf{q}_{j} \mathbf{r}+\varphi_{j}\right) \rho(U(\mathbf{r})) d^{3} \mathbf{r} .
$$

Here trivial factor $\Delta n_{\max }$ is omitted. Normalization coefficient $2 / V$, with $V$ being integration volume, is chosen in such a way, that for first five curves of saturation $\rho_{\alpha}(U)$ the value of $F_{j}$ is equal to $U_{j}$ at $N U_{1} \ll 1$. In actual calculations we used onedimensional integral over the interval length $L$, with normalization coefficient $2 / L$ :

$$
F_{j}=\frac{2}{L} \int_{0}^{L} \cos \left(q_{j} x+\varphi_{j}\right) \rho(U(x)) d x .
$$


The values of $q_{j} L$ were around 100 radian and more, $q_{j}$ were chosen mutually non-commensurate, and phases $\varphi_{j}$ were random within interval $(0,2 \pi)$. We successfully checked that if some particular component $U_{k}\left[1+\cos \left(q_{k} x+\varphi_{k}\right)\right]$ was absent during recording, then the Fourier-component $F_{k}$ calculated by Eq. (13) was much smaller than those $F_{j}$, whose $U_{j}\left[1+\cos \left(q_{j} x+\varphi_{j}\right)\right]$ were actually present at recording.

\section{Results for recording of single VBG}

Our first step was to find the dependence of Fourier component of $\delta n(\mathbf{r})$ for recording of a single grating: $N=1$, on the value of exposure $N U_{1} \equiv U_{1}$. Figure 3 shows the dependence of $F_{1}$ on $U_{1}$ for four out of seven studied curves of saturation: $\tanh (U)$, $(2 / \pi) \arctan (\pi U / 2)$, straight linear between $(U=0, \rho=0)$ and $(U=1, \rho=1)$ and step-function from 0 to 1 at $U=0.5$. All 7 studied laws of saturation have demonstrated a peak of $F_{1}$ around $U_{1} \sim 1$ and a decrease of $F_{1}$ for large $U_{1}$. To eliminate clutter on the graph, we depicted only four curves corresponding to Eqs. (1), (2), (5), (7). The peak values of $F_{1}$ peak and corresponding exposure $U_{1}$ delivering that $F_{1}$ peak for all seven saturation laws are presented in the first pair of lines of Table 1.

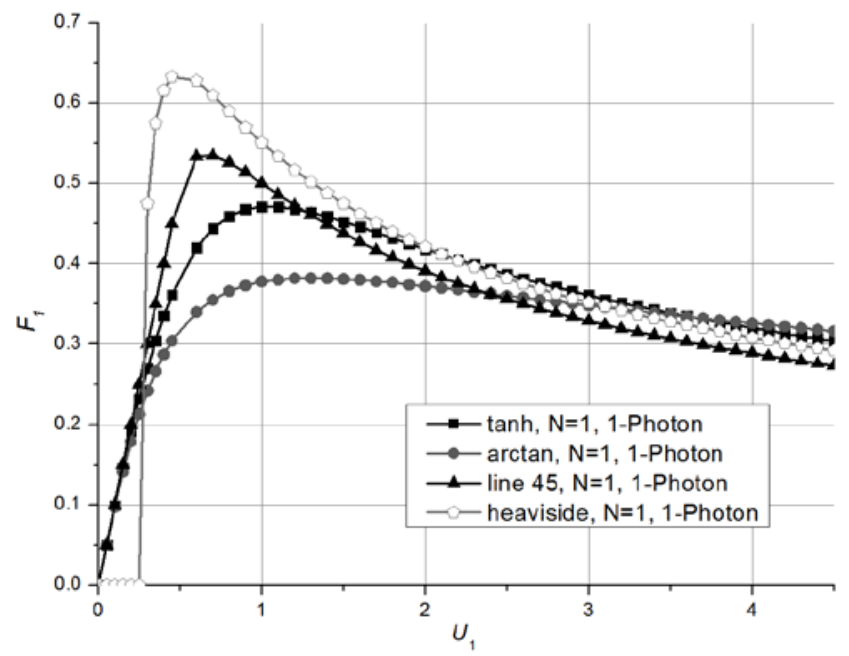

Fig. 3. Dependence of Fourier amplitude $F_{j}$ of a single recorded grating on average exposure $U_{1}$ for different laws of saturation in case of one-photon absorption.

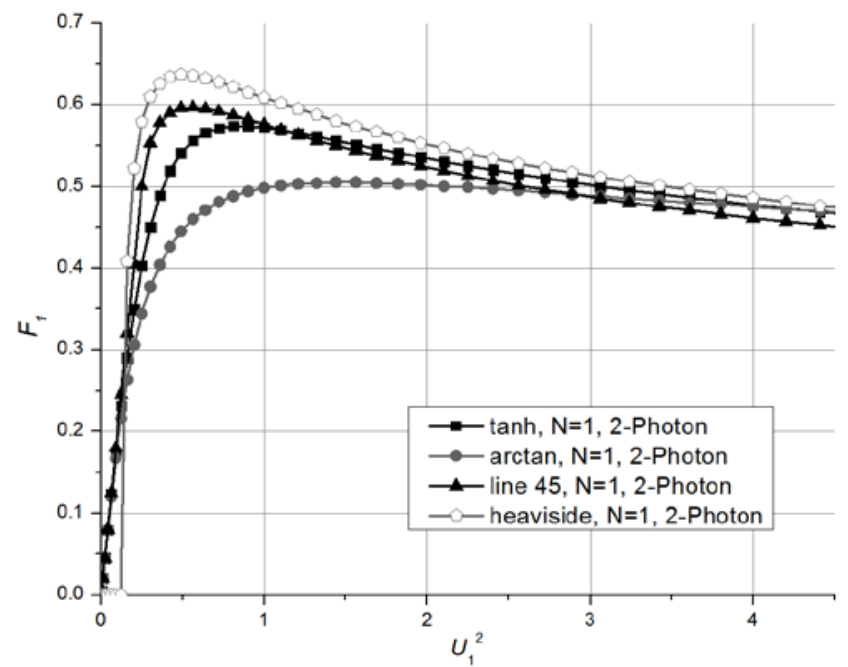

Fig. 4. Same graph as on Fig.3, but for the case of two-photon absorption.
Qualitative conclusion is that sharper $\rho(U)$ curves yield larger values of $F_{1 \text {,peak }}$. However, difference between largest $F_{1 \text {,peak }}$ for $\rho_{\text {heaviside }}(U)$ and smallest $F_{1 \text {,peak }}$ for power law saturation constitutes only factor around 1.85. Curves at Figure 3 and data at Table 1 were produced for single-photon absorption model of recording. Corresponding data for two-photon absorption model of recording are presented at Figure 4 and Table 2.

\section{Multiplexed VBG with $N=4,8,16,32$ and 64 gratings}

Figure 5 shows the average Fourier component $\left\langle F_{j}\right\rangle$ for $j=1,2,3,4$ at single-photon recording of $N=4$ independent gratings, and Figure 7 shows same results for $N=32$ independent gratings. Again, as on Figure 3, we tried not to overburden the graph keeping the curves for four laws of saturation only. Argument on horizontal axis is spatiallyaveraged exposure $U_{\mathrm{av}}=N U_{1}$. Table 1 gives the peak values of average Fourier component $F_{j}$ (peak) and spatially average exposure $N U_{j} \equiv N U_{1}$ delivering that peak. Figures 6,8 and Table 2 provide similar information for model of two-photon absorption.

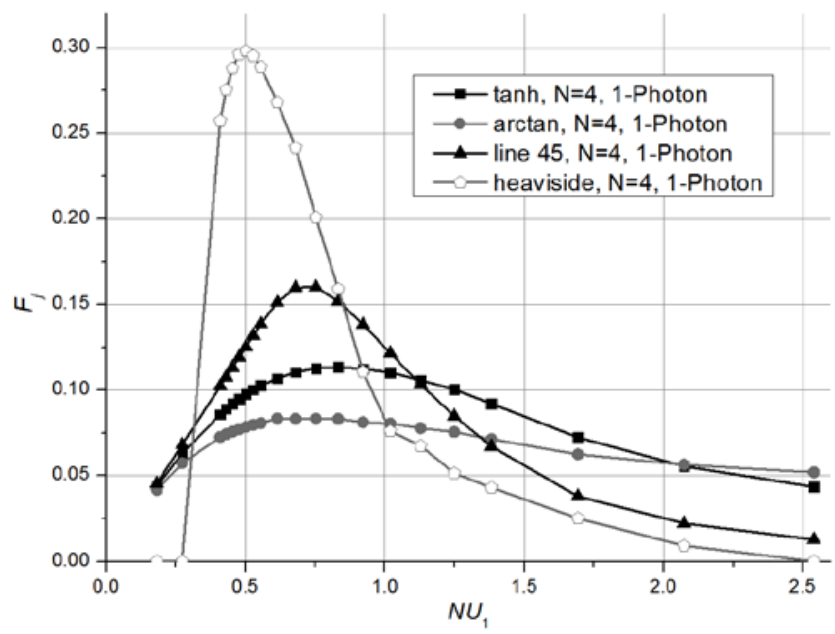

Fig. 5. Dependence of Fourier amplitude $F_{j}$ of $N=4$ grating on average exposure $\mathrm{NU}_{1}$ for various laws of saturation in case of one-photon absorption.

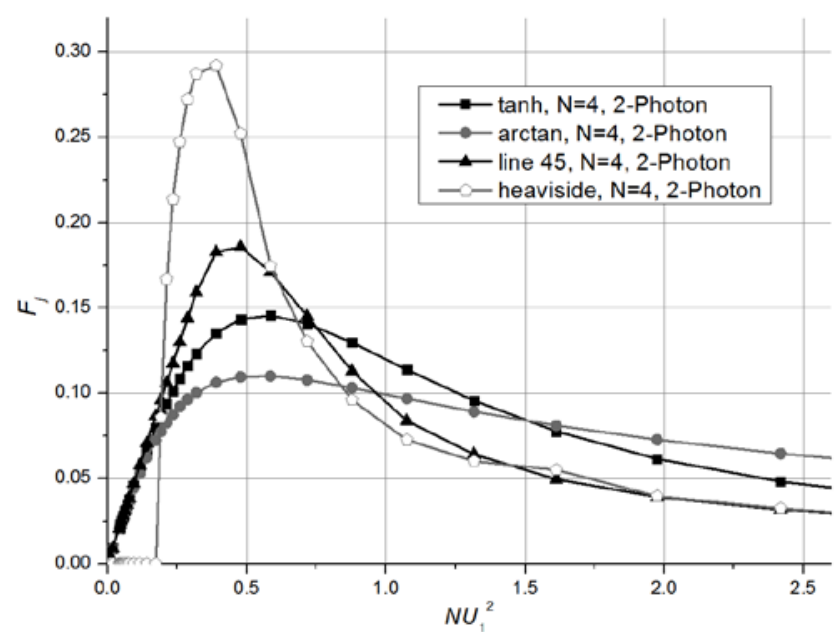

Fig. 6. Same graph as on Fig.5, but for the case of two-photon absorption 


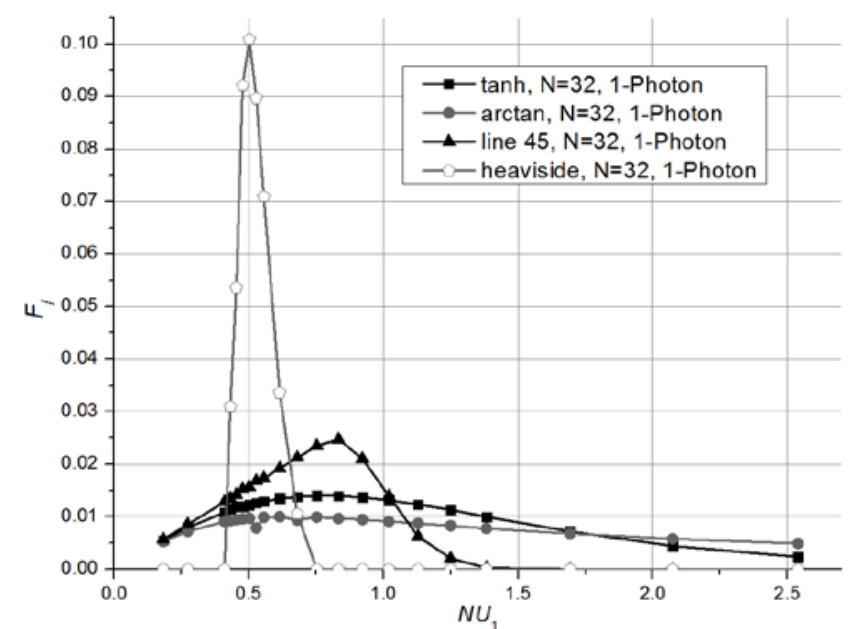

Fig. 7. Dependence of Fourier amplitude $F_{j}$ of $N=32$ gratings on average exposure $N U_{1}$ for different laws of saturation in case of one-photon absorption.

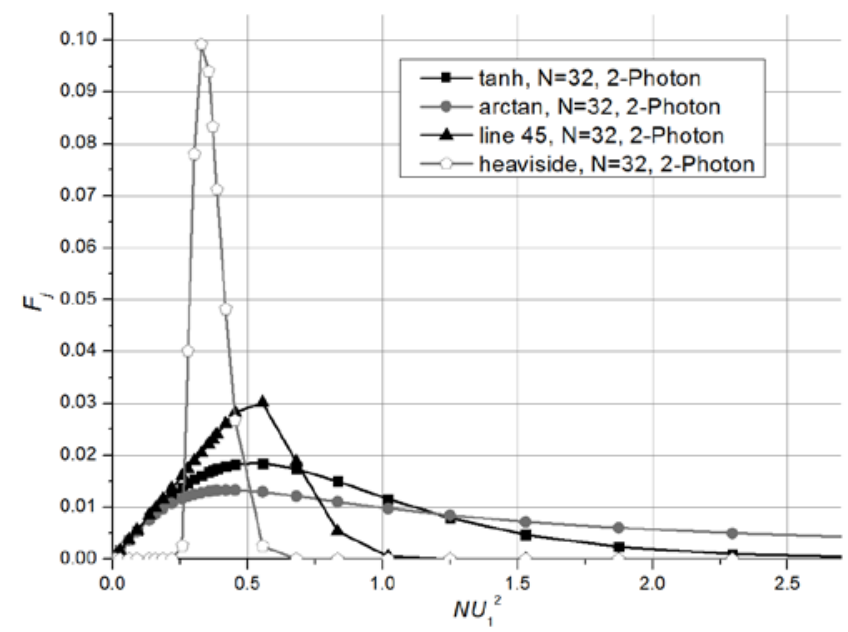

Fig. 8. Same graph as on Fig.7, but for the case of two-photon absorption.

\section{Dependence of peak Fourier amplitude $F_{j}$ on multiplexity $N$ : analytic calculations and numerical modeling}

Analytic calculations for a large number of individually recorded gratings may be done via decomposition $U(\mathbf{r})=N U_{1}+\sum_{j=1}^{N} U_{1} \cos \left(\mathbf{q}_{j} \mathbf{r}+\varphi_{j}\right)$ for single-photon case, so that

$$
\begin{gathered}
n(x)=\Delta n_{\max }\left[\rho(\xi)+\frac{\partial \rho}{\partial \xi} U_{1} \cos \left(q_{j} x+\varphi_{j}\right)\right], \quad \xi=N U_{1}, \\
F_{j}=\frac{\partial \rho}{\partial \xi} U_{j} \equiv \frac{1}{N} \xi \frac{\partial \rho}{\partial \xi^{.}}
\end{gathered}
$$

The values of maximum of functions $\xi \partial \rho / \partial \xi$ are $\left[\xi \partial \rho_{\tanh } / \partial \xi\right]_{\max }=0.448, \quad\left[\xi \partial \rho_{\arctan } / \partial \xi\right]_{\max }=1 / \pi=0.38$, $\left[\xi \partial \rho_{\text {power }} / \partial \xi\right]_{\max }=1 / 4=0.25, \quad\left[\xi \partial \rho_{\exp } / \partial \xi\right]_{\max }=e^{-1}=$ $0.368, \quad\left[\xi \partial \rho_{\text {line } 45} / \partial \xi\right]_{\max }=1,\left[\xi \partial \rho_{\text {line } 60} / \partial \xi\right]_{\max }=2$ at $\xi_{\text {tanh }}=0.776, \xi_{\text {arctan }}=2 / \pi=0.637, \quad \xi_{\text {power }}=1, \xi_{\exp }=1$, $\xi_{\text {line } 45}=1, \quad \xi_{\text {line } 60}=1$, respectively.

Results of numerical modeling yield at least for $N \geq 4$ reasonable agreement with Eq. (14).

Special attention should be paid to the case of Heaviside function, $\rho_{\text {heaviside }}(\xi)$ at $\xi=0.5$. The optimal value of $N U_{1}=\xi_{\text {opt }}$ is evidently $\xi_{\text {opt, heaviside }}=0.5$, so that $U_{1 \text { opt, heaviside }}=0.5 / N$.

After that one should consider $u(x) \approx 0.5+r+m(x)$. Here $m(x)=U_{1} \cos \left(q_{1} x\right)$, and $r$ is random quantity, which is the result of adding all spatially-oscillating parts of all other remaining sinusoidal profiles of recording: so to say other recorded gratings. Probability distribution $W(r) d r$, due to central limit theorem, is well approximated by Gaussian, $W(r) d r \approx\left(2 \pi \sigma^{2}\right)^{-0.5} \exp \left(-r^{2} / 2 \sigma^{2}\right) d r$, where $\sigma^{2} \approx 0.5 N U_{j}^{2}$. With account of optimum $U_{j}=0.5 / N$, square of standard deviation for $r$ becomes $\sigma^{2} \approx 1 /(8 N)$. It means that the value of $\rho_{\mathrm{st}}(0.5+r+m(x))$, averaged over fluctuations of $r$, approximately equals $W(0) m(x)=m(x) \sqrt{4 N / \pi}$. Further spatial averaging of $\cos ^{2}\left(q_{1} x\right)$ yields factor 0.5 , and with account of $U_{1} \approx 0.5 / N$ one gets the optimum multiplexed grating for Heaviside response of multiplexed VBG $F_{j} \approx 1 / \sqrt{\pi N}$.

This result is also in reasonable agreement with numerical modelling at $N \gg 1$.

In case of two-photon recording the value of optimum $\xi$ for different $\rho(U)$ curves should be equalized to $N \cdot\left(3 U_{1}{ }^{2} / 2\right)$ and

$$
F_{j}=2 U_{1}^{2}\left(\xi \frac{d \rho}{d \xi}\right)_{\text {opt }}=\frac{4}{3}\left(\xi_{\text {opt }} \frac{d \rho}{d \xi}\right) \frac{1}{N}, \quad U_{1}^{2}=\frac{2 \xi_{\text {opt }}}{3 N} .
$$

And again, numerical modeling at $N \gg 1$ is in a reasonable agreement with this extra factor $4 / 3$ for two-photon recording.

Quite interesting is the problem of cross-modulation gratings. Namely, if recording profile contains, among others, the terms

\begin{tabular}{|c|c|c|c|c|c|c|c|c|}
\hline $\mathrm{N}$ & & tanh & $\arctan$ & power & $\exp$ & line 45 & line 60 & heaviside \\
\hline 1 & $F_{1}$ peak & 0.472 & 0.382 & 0.343 & 0.438 & 0.536 & 0.625 & 0.637 \\
\hline 4 & $F_{1}$ peak & 0.113 & 0.083 & 0.068 & 0.097 & 0.160 & 0.267 & 0.298 \\
\hline \multirow{2}{*}{8} & $F_{1}$ peak & 0.056 & 0.041 & 0.033 & 0.047 & 0.086 & 0.158 & 0.199 \\
\hline & $N \cdot U_{1}$ for peak value & 0.833 & 0.680 & 1.129 & 1.129 & 0.752 & 0.752 & 0.502 \\
\hline 16 & $F_{1}$ peak & 0.028 & 0.020 & 0.016 & 0.023 & 0.046 & 0.090 & 0.142 \\
\hline 32 & $N \cdot U_{1}$ for peak value & 0.752 & 0.614 & 1.249 & 1.020 & 0.833 & 0.833 & 0.502 \\
\hline \multirow{2}{*}{64} & $F_{1}$ peak & 0.007 & 0.006 & 0.004 & 0.007 & 0.013 & 0.026 & 0.072 \\
\hline & $N \cdot U_{1}$ for peak value & 0.462 & 0.566 & 0.502 & 0.752 & 0.833 & 0.833 & 0.502 \\
\hline
\end{tabular}
$U_{j}\left(1+\cos \left(q_{j} x+\varphi_{j}\right)\right)$ and $U_{k}\left(1+\cos \left(q_{k} x+\varphi_{k}\right)\right)$, then a

Table 1. Peak Fourier amplitudes for all seven functions for $N=1,4,8,16,32$ and 64 gratings in case of one-photon absorption. 
Table 2. Peak Fourier amplitudes for all seven functions for $N=1,4,8,16,32$ and 64 gratings in case of two-photon absorption.

\begin{tabular}{|c|c|c|c|c|c|c|c|c|}
\hline $\mathrm{N}$ & & $\tanh$ & $\arctan$ & power & $\exp$ & line 45 & line 60 & heaviside \\
\hline 1 & $F_{1}$ peak & 0.574 & 0.505 & 0.476 & 0.556 & 0.597 & 0.633 & 0.637 \\
\hline 4 & $F_{1}$ peak & 0.145 & 0.110 & 0.092 & 0.127 & 0.186 & 0.272 & 0.292 \\
\hline \multirow{2}{*}{8} & $F_{1}$ peak & 0.074 & 0.054 & 0.044 & 0.062 & 0.104 & 0.173 & 0.198 \\
\hline & $N \cdot U_{1}$ for peak value & 0.537 & 0.476 & 0.658 & 0.658 & 0.476 & 0.537 & 0.358 \\
\hline 16 & $F_{1}$ peak & 0.037 & 0.028 & 0.021 & 0.030 & 0.056 & 0.104 & 0.139 \\
\hline 32 & $N \cdot U_{1}$ for peak value & 0.555 & 0.453 & 0.680 & 0.680 & 0.555 & 0.555 & 0.328 \\
\hline \multirow{2}{*}{64} & $F_{1}$ peak & 0.009 & 0.006 & 0.005 & 0.008 & 0.016 & 0.033 & 0.069 \\
\hline & $N \cdot U_{1}$ for peak value & 0.510 & 0.434 & 0.706 & 0.706 & 0.553 & 0.553 & 0.327 \\
\hline
\end{tabular}

grating

$$
\begin{aligned}
\delta n(x)=\Delta & n_{\max } \cdot F_{j k} \cdot\left\{\cos \left[\left(q_{j}+q_{k}\right) x+\varphi_{j}+\varphi_{k}\right]+\right. \\
& \left.+\cos \left[\left(q_{j}-q_{k}\right) x+\varphi_{j}-\varphi_{k}\right]\right\}
\end{aligned}
$$

is recorded. Again, at $N \gtrsim 4$ analytic expansion of $\rho(U)$ around $\langle U\rangle=N U_{1}$ allows to predict

$$
F_{j k} \approx \frac{1}{2 N^{2}}\left[U^{2} \frac{d^{2} \rho(U)}{d U^{2}}\right]_{U=\langle U\rangle=N U_{1}} .
$$

So the amplitude of "parasitic" cross-modulation gratings decreases as $1 / N^{2}$, if spatial average exposure $U_{1} N$ is chosen to optimize the amplitudes of main gratings. Our numerical modelling with random phases $\varphi_{j}$ and $\varphi_{k}$ is in a good agreement with analytical expression (17).

\section{Conclusion}

We have studied theoretically the recording of multiplexed Volume Bragg Gratings with account of seven possible shapes of saturation curves. Optimum values of spatially averaged total exposure were found for each of those saturation curves, and the corresponding Fourier amplitudes of individual gratings. For relatively large multiplicity, number of gratings $N \gtrsim 4$, analytical formulae (14-16) derived by us, are in a good correspondence with the results of numerical modeling. In particular, best amplitude of individual Fourier component goes down as const $/ N$, and values of that const are determined for each normalized saturation curve $\rho(U)$ on exposure $U$.

Qualitative conclusion is that sharper profiles of saturation curve $\rho(U)$ yield larger Fourier amplitudes at optimum exposure. Especially good would be threshold-like profile $\rho(U)$ of Heaviside function. Similar beneficial effect shows at sharper saturation due to two-photon recording. However, the price one should pay for this sharpness-provided advantage is the necessity of more precise adjustment of spatially-averaged exposure/development.

Acknowledgments. The work was supported by HEL JTO contract W911NF-10-1-0441.

\section{References}

1. L. Glebov, "Volume Holographic Elements in a PhotoThermo-Refractive Glass," J. Holography and Speckle 5, 77 (2009).

2. J. Lumeau and L. B. Glebov, "Effect of the refractive index change kinetics of photosensitive materials on the

diffraction efficiency of reflecting Bragg gratings," Appl. Opt. 52, 3993-3997 (2013).

3. A. Sevian, O. Andrusyak, I. Ciapurin, V. Smirnov, G. Venus, and L. Glebov, "Efficient power scaling of laser radiation by spectral beam combining," Opt. Lett. 33, 384386 (2008).

4. O. Andrusyak, V. Smirnov, G. Venus, V. Rotar, and L. Glebov, "Spectral Combining and Coherent Coupling of Lasers by Volume Bragg Gratings," IEEE J. Sel. Topics Quantum Electron. 15, 344-353 (2009).

5. D. Ott, I. Divliansky, B. Anderson, G. Venus, and L. Glebov, "Scaling the spectral beam combining channels in a multiplexed volume Bragg grating," Opt. Express 21, 29620-29627 (2013).

6. G. Chang, M. Rever, V. Smirnov, L. Glebov, and A. Galvanauskas, "Femtosecond Yb-fiber chirped-pulseamplification system based on chirped-volume Bragg gratings," Opt. Lett. 34, 2952-2954 (2009).

7. S. Kaim, S. Mokhov, B. Ya. Zeldovich, and L. B. Glebov, "Stretching and compressing of short laser pulses by chirped volume Bragg gratings: analytic and numerical modeling," Opt. Eng. 53, 051509-7 (2014).

8. G. B. Venus, A. Sevian, V. Smirnov, and L. B. Glebov, "High-brightness narrow-line laser diode source with volume Bragg-grating feedback," Proc. SPIE 5711, 166176 (2005).

9. S. Mokhov, A. Jain, C. Spiegelberg, V. Smirnov, O. Andrusyak, G. Venus, B. Zeldovich, and L. Glebov, "Multiplexed Reflective Volume Bragg Grating for Passive Coherent Beam Combining," in Frontiers in Optics 2010/Laser Science XXVI, (OSA, 2010), paper LWG2.

10. V. Smirnov, J. Lumeau, S. Mokhov, B. Ya. Zeldovich, and L. B. Glebov. Ultranarrow bandwidth moiré reflecting Bragg gratings recorded in photo-thermo-refractive glass. Opt. Lett. 35, 592-594 (2010).

11. A. L. Glebov, O. Mokhun, A. Rapaport, S. Vergnole, V. Smirnov, L. B. Glebov. Volume Bragg gratings as ultranarrow and multiband optical filters. Micro-Optics 2012, edited by H. Thienpont, J. Mohr, H. Zappe, H. Nakajima. Proc. of SPIE Vol. 8428, 84280C, 1-11 (2012).

12. L. Solymar, "Two-dimensional $N$-coupled-wave theory for volume holograms," Opt. Comm. 23, 199-202 (1977).

13. D.Psaltis, D. Brady, K.Wagner, "Adaptive optical networks using photorefractive crystals," Applied Optics V. 27, 1752-1759 (1988).

14. J.R.Wullert II, Y.Lu, "Limits of the capacity and density of holographic storage," Applied Optics V. 33, 2192-2196 (1994).

15. P. Günter, J. P. Huignard, Photorefractive Materials and Their Applications 1: Basic Effects v.2, (Springer, 2005). 